\title{
EDITORIAL
}

\section{Editorial zum Schwerpunktthema: Schulleitungsfortbildung - Stand und Desiderate}

\section{Editorial to the Focus Topic: \\ Principals' Training - Current State and Desiderata}

Seit den 1990er-Jahren hat sich das Aufgabenportfolio von Schulleiter ${ }^{*}$ innen entscheidend verändert. Bestand ihre Aufgabe zuvor vor allem darin, die Funktionalität ihrer Schule zu gewährleisten, wird nun erwartet, dass sie als Manager*innen und Schulentwickler*innen ihre Schulen gestalten und dabei in der Organisations-, Personal- und Unterrichtsentwicklung Führungsaufgaben übernehmen. Für diese Aufgaben benötigen sie spezifisches Expertenwissen und Kompetenzen, die sie nicht im Rahmen der ersten und zweiten Phase der Lehrerbildung erwerben (Buchen \& Rolff, 2016). Inzwischen haben die meisten Bundesländer eine verpflichtende Erstausbildung für (angehende) Schulleiter*innen eingerichtet (Tulowitzki, Hinzen \& Roller, 2019). Zugleich zeigen allerdings aktuelle Studien, dass ein Großteil der Schulleiter*innen vor Antritt der Tätigkeit keine spezifische Qualifizierung für die Schulleitung erhalten hat (z. B. Schwanenberg, Klein \& Walpuski, 2018).

Von der veränderten Qualifizierung profitieren vor allem Schulleiter*innen, die in den letzten Jahren das Amt angetreten haben. Es überrascht nicht, dass Schulleitungen sich insbesondere durch Schulentwicklungsaufgaben belastet fühlen und Fortbildungsund Unterstützungsbedürfnisse gerade in diesem Bereich berichten (Brauckmann \& Herrmann, 2013; Cramer, Pietsch, Tulowitzki \& Groß Ophoff, 2020; Schwanenberg et al., 2018). Dementsprechend ist inzwischen durch verschiedene Studien dokumentiert, dass Schulleiter*innen in Deutschland in hohem Maße an Fortbildungsangeboten teilnehmen, die sich vor allem auf Themen der Schulentwicklung beziehen (Schwanenberg et al., 2018), und dabei neben staatlichen Fortbildungsangeboten beispielsweise auch alternative Formate zur Führungskräfteentwicklung, wie etwa Schulleitungsnetzwerke oder Coaching-Angebote, nutzen (Cramer et al., 2020).

Vor diesem Hintergrund hat die Fortbildung von Schulleiter*innen eine zentrale Bedeutung. Umso bemerkenswerter ist, dass sie bislang einen blinden Fleck in der deutschsprachigen Schulleitungsforschung darstellt. Dieses lässt sich durchaus als Ausdruck einer fehlenden Klärung der Unterstützungsbedarfe von Schulen 
im Kontext der Schulentwicklung lesen: Während im deutschsprachigen Schulentwicklungsdiskurs der Fokus vor allem auf der Eigenverantwortung der Schulen liegt, werden die Unterstützungsstrukturen, die Schulen benötigen, um diese Verantwortung tatsächlich übernehmen zu können - zu denen auch eine systematische Führungskräfteentwicklung gehört -, auch nach mehr als zwei Jahrzehnten Schulentwicklungsdiskurs eher stiefmütterlich behandelt (Böttcher \& Luig, 2020; Klein, im Erscheinen).

Das vorliegende Heft greift dieses Desiderat auf, indem ein Überblick über das Praxisund Forschungsfeld gegeben, die Wirkung einer Schulleitungsfortbildung analysiert und ein Einblick in die Praxis der Schulleitungsfortbildung geboten werden.

Im Beitrag von Esther Dominique Klein und Pierre Tulowitzki wird dazu zunächst der Versuch unternommen, einen Überblick über das Themenfeld „Schulleitungsfortbildung“ zu verschaffen. Die Autor*innen zeigen auf, dass es zwar eine Fülle an Fortbildungsangeboten für Schulleiter*innen gibt, aber bislang kaum eine vertiefte theoretische Auseinandersetzung mit Lernfeldern für die Führungskräfteentwicklung, keine systematische Bestandsaufnahme von Fortbildungsformaten und -anbietern und kaum Forschung zu den Wirkungen der Schulleitungsfortbildung. Die Autor ${ }^{\star}$ innen verweisen sowohl auf die Herausforderungen an die Schulleitungsforschung, dieses Themenfeld stärker in den Fokus zu rücken, als auch auf die Verantwortung für die Schulaufsicht, Fortbildungsangebote stärker mit systematischen Feedbackstrukturen zu verknüpfen.

Der Beitrag von André Meyer, Eric Richter, Anna Gronostaj und Dirk Richter geht auf eine großflächig angelegte Schulleitungsfortbildung zur Schulentwicklung im Saarland ein. Die Fortbildung Werkstatt „Schule leiten“ wurde durch die Deutsche Schulakademie entwickelt und bereits in mehreren Durchgängen in dem Bundesland durchgeführt. Im vorliegenden Beitrag werden erste Ergebnisse der Begleitforschung berichtet, und die Autor*innen gehen darauf ein, wie sich ausgewählte Aspekte des Führungsverhaltens im Verlauf der Fortbildung verändern. Darüber hinaus nehmen die Autor*innen in den Blick, ob sich die festgestellten Veränderungen für bestimmte Personengruppen unterscheiden. Dabei wird deutlich, dass in Analysen zu den Effekten einer Fortbildung neben den mittleren Veränderungen auch die individuellen Verläufe von Relevanz sind.

Während das Fortbildungsangebot für Schulleitungen in Deutschland einem Flickenteppich gleicht, zeigt ein Blick in die deutschsprachigen Nachbarländer, dass es dort durchaus stringenter organisierte Ansätze gibt. Robert Pham-Xuan und Markus Ammann stellen in ihrem Bericht Neuerungen in der Schulleitungsfortbildung aus Österreich vor und verweisen vor diesem Hintergrund darauf, dass diese gesetzlich reguliert und durch feste Anbieter erbracht wird. Niels Anderegg und Johannes Breitschaft berichten, dass die Schulleitung in der Schweiz eine „junge Profession“ sei; 
trotzdem - oder gerade deswegen - findet sich aber auch hier eine Systematisierung der Schulleitungsfortbildung durch deren Verankerung an den Pädagogischen Hochschulen.

Dass eine solche Verankerung im Hochschulwesen in Deutschland bislang kaum vorliegt, führt der Beitrag von Michael Krüger, in dem Schulmanagement-Studiengänge an Hochschulen näher betrachtet werden, vor Augen. Die Arbeit stellt die Entwicklung der Studiengänge an deutschen Hochschulen dar und beleuchtet die inhaltliche Ausrichtung der Programme.

Zuletzt zeigen Cornelia von Ilsemann und Wilfried Kretschmer aus der Perspektive von Fortbildner*innen auf, dass die Fortbildung von Schulleiter ${ }^{\star}$ innen ohnehin vielschichtiger $\mathrm{zu}$ gestalten ist und diversen Anforderungen gerecht werden muss, die Kursangebote an Hochschulen nur bedingt erfüllen können. Diese Arbeit basiert auf Erfahrungen, die im Rahmen der Durchführung der Werkstatt „Schule leiten“ gesammelt wurden.

Die Rolle von Schulleitungen ist in diesen Zeiten eine besonders herausfordernde und umso bedeutsamere Tätigkeit im Hinblick auf die Funktionsfähigkeit und die Weiterentwicklung der Organisation Schule mit hybriden Lehr- und Lernräumen. An das Schulleitungsthema schließen auch Pierre Tulowitzki und Julia Gerick mit ihrem Bericht an; sie präsentieren empirische Befunde zur Schulleitung in der digitalisierten Welt.

Der Diskussionsbeitrag von Christian Timo Zenke „Vom Klassenzimmer zur Lernlandschaft?" Über eine Expedition ins Ungewisse" ist schließlich anschlussfähig an eine räumlich entgrenzte Situation von Schule.

Esther Dominique Klein, Dirk Richter \& Isabell van Ackeren

\section{Literatur und Internetquellen}

Böttcher, W., \& Luig, C. (2020). Schulaufsicht als Managementaufgabe. In E. D. Klein \& N. Bremm (Hrsg.), Unterstützung - Kooperation - Kontrolle. Zum Verhältnis von Schulaufsicht und Schulleitung in der Schulentwicklung (S. 107-124). Wiesbaden: Springer VS. https://doi.org/10.1007/978-3-658-28177-9_6

Brauckmann, S., \& Herrmann, C. (2013). Belastungserleben von Schulleiterinnen und Schulleitern im Rahmen erweiterter schulischer Eigenständigkeit. Erste empirische Befunde aus der SHaRP-Studie. In I. van Ackeren, M. Heinrich \& F. Thiel (Hrsg.), Evidenzbasierte Steuerung im Bildungssystem. Befunde aus dem BMBF-SteBis-Verbund (DDS - Die Deutsche Schule, 12. Beiheft) (S. 172-197). Münster: Waxmann.

Buchen, H., \& Rolff, H.-G. (Hrsg.). (2016). Professionswissen Schulleitung (4., überarb. u. erw. Aufl.). Weinheim: Beltz. 
| Editorial

Cramer, C., Pietsch, M., Tulowitzki, P., \& Groß Ophoff, J. (2020). Schulleitungen in Deutschland - Kurzbericht zur Studie. Zugriff am 07.07.2020. Verfügbar unter: https:// osf.io/vwsc7/.

Klein, E. D. (im Erscheinen). Von Steuerungsverantwortung und Stolpersteinen - Die Schulaufsicht als Unterstützungsinstanz für Schulentwicklung. In T. Webs \& V. Manitius (Hrsg.), Unterstützungssysteme für Schulen. Bielefeld: wbv.

Schwanenberg, J., Klein, E. D., \& Walpuski, M. (2018). Wie erfolgreich fühlen sich Schulleitungen und welche Unterstützungsbedürfnisse haben sie? Ergebnisse aus dem Projekt Schulleitungsmonitor (SHIP Working Paper Reihe, No. 03). Essen: Universität Duisburg-Essen. https://doi.org/10.17185/duepublico/47202

Tulowitzki, P., Hinzen, I., \& Roller, M. (2019). Qualifizierung von Schulleiterinnen in Deutschland - ein bundesweiter Überblick. DDS - Die Deutsche Schule, 111 (2), 149169. https://doi.org/10.31244/dds.2019.02.04

https://doi.org/10.31244/dds.2020.03.01 